\title{
Effect of SNPs in genes regulating cell cycle control, apoptosis and inflammation on lung cancer susceptibility of northern Thai population
}

\author{
Rattanasak Wongkomonched ${ }^{\mathrm{a}, \mathrm{b}}$, Bruce Rannala ${ }^{\mathrm{c}}$, Phongtape Wiwatanadate ${ }^{\mathrm{d}}$, Somchareon Saeteng ${ }^{\mathrm{e}}$, \\ Jatupol Kampuansai ${ }^{\mathrm{a}, \mathrm{f}, *}$, Daoroong Kangwanpong ${ }^{\mathrm{a}}$ \\ a Department of Biology, Faculty of Science, Chiang Mai University, Chiang Mai 50200 Thailand \\ b PhD Program in Biology, Faculty of Science, Chiang Mai University, Chiang Mai 50200 Thailand \\ c UC Davis Genome Center, University of California Davis, CA 95616 USA \\ d Department of Community Medicine, Faculty of Medicine, Chiang Mai University, Chiang Mai 50200 \\ Thailand \\ e Department of Surgery, Faculty of Medicine, Chiang Mai University, Chiang Mai 50200 Thailand \\ ${ }^{\mathrm{f}}$ Research Center in Bioresources for Agriculture, Industry and Medicine, Chiang Mai University, Chiang \\ Mai 50200 Thailand
}

*Corresponding author, e-mail: Jatupol.K@cmu.ac.th

\begin{abstract}
Northern Thai population has experienced a high incidence of lung cancer and greater rates of mortality over the past several decades. In this study, blood samples and personal information were collected from 91 non-small cell lung cancer patients and 84 cancer-free healthy unrelated volunteers living in northern Thailand. Eight functional SNPs, including cell cycle control (TP53 Pro72Arg, MDM2 T309G, CCND1 G723A, CDKN1A Ser31Arg), apoptosis (FASLG C-844T, FAS G-1378A) and inflammation (TGFB1 T-1347C, TGFB1 Pro10Leu) were genotyped by multiplex allelespecific polymerase chain reactions. Odds ratios (OR) with $95 \%$ confidence intervals (CI) were calculated using logistic regression after making adjustments for age, gender and the smoking status of all participating subjects. No significant association between a single SNP and lung cancer risk was observed. However, a combination of TGFB1-1347T and 10Pro allele carriers indicated a significantly increased risk for lung cancer ( $\mathrm{OR}=2.90,95 \% \mathrm{CI}=1.01-8.4, \mathrm{P}=0.049$ ) relative to wild-type genotypes. Among the participants, who had kitchens inside their homes, -1347T allele carriers had significant increase in lung cancer risk $(\mathrm{OR}=5.9,95 \% \mathrm{CI}=1.32-26.5, \mathrm{P}=0.020)$ when compared to the TGFB1$1347 \mathrm{C} / \mathrm{C}$ genotype carriers. In conclusion, we have observed a significant association between TGFB1-1347T allele and either TGFB1 10Pro allele or having kitchen inside the house on risk elevation of non-small cell lung cancer among the population of northern Thailand.
\end{abstract}

KEYWORDS: lung cancer, northern Thailand, genetic susceptibility

\section{INTRODUCTION}

Lung cancer has been the most common form of diagnosed cancer worldwide and has been associated with the highest rates of global incidence and mortality according to the International Agency for Research on Cancer (IARC) in a 2018 report [1]. In Thailand, lung cancer ranked second in mortality rate among Thai males, preceded by liver cancer in 2015. Lung cancer has high incidence in the north (especially the three provinces: Chiang Mai, Lampang and Lamphun) while liver cancer is extremely high in the north-east $[2,3]$. Based on the most recent volume of the Cancer in Thailand, the annual age-standardized incidence rates per 100000 (for the years 2013-2015) of lung cancer for males and females, respectively, were 32.1 and 21.6 in Chiang Mai, 32.9 and 19.5 in Lampang and 37.9, and 19.8 in Lamphun [3]. Most of these patients were found to have an advanced stage of lung cancer that was either diagnosed as regional or as distant metastases. Non-small cell lung cancer was found to be the most common type of lung cancer with adenocarcinoma representing more than half of the total number of incidences [3]. Notably, more than $80 \%$ of the lung cancer patients died within one year after being diagnosed [4]. This evidence clearly suggests that lung cancer is a major health issue for 
the population of northern Thailand.

The etiology of lung cancer among the northern Thai population has been associated with exposure to carcinogens stemming from personal habits, occupations and the environment along with certain inherited genetic factors. In accordance with the high prevalence $(34.2 \%)$ of daily smoking habits among those residing in the northern part of Thailand from 1991 to 2007 [5], tobacco smoking has been identified as the most significant risk factor for lung cancer in this region. Environmental factors, including residential radon [6], outdoor air pollution originating from vegetative burning, seasonal bushfires, engine exhaust emissions $[7,8]$, and indoor air combustion from the burning of biomass fuels [9], also contributed to the accelerated development of lung cancer among the northern Thai population. An increased risk of TP53 somatic mutations in lung tissues was found among individuals who used pesticides, had kitchens inside their homes, or lived in concrete homes in comparison with those who were not exposed to any of these risk factors [10]. Recently, a significant association between a high level of indoor radon in the household and a short telomere length has been observed [11]. Interestingly, a combination of genetic polymorphisms within five genes was found to be involved with carcinogen metabolism and DNA repair, and has been significantly associated with an elevated risk of developing lung cancer among northern Thai women [12].

Effects of genetic variations, especially with regard to single nucleotide polymorphisms (SNPs) on the susceptibility to lung cancer, were depended on their location within the genes and the role of proteins encoded from those genes. Previous studies have reported that the SNPs found in genes regulating p53-mediatied cell cycle control (i.e. TP53 Pro72Arg, MDM2 T309G, CCND1 G723A, CDKN1A Ser31Arg) [13-15], apoptosis (i.e. FASLG C-844T, FAS G-1378A) [16] and inflammation (i.e. TGFB1 T-1347C, TGFB1 Pro10Leu) [17] could affect individual lung cancer susceptibility in Asians. Combinations of these SNPs were also found to be significantly related to an increased risk of developing lung cancer in some studies $[15,16]$. In the absence of a smoking habit, several SNPs in these gene groups were significantly associated with an elevated risk of developing lung adenocarcinoma among female non-smokers, who were exposed to certain environmental factors, including cooking oil fumes, fuel smoke and environmental tobacco smoke $[18,19]$.
This study aimed to evaluate the association between candidate SNPs in genes regulating cell cycle control, apoptosis and inflammation with the risk of developing lung cancer among the population of northern Thailand. Subgroup analyses, involving combinations of two SNPs in the same gene group and individuals exposed to each of the potential environmental risk factors for lung cancer, were also performed.

\section{MATERIALS AND METHODS}

\section{Ethics statement}

During the course of our research, we protected the rights of the participants along with their identities. We confirmed that all experiments were performed in accordance with the relevant guidelines and regulations established for the experimental protocol on human subjects, as has been approved by the Research Ethics Committee 3, Faculty of Medicine, Chiang Mai University, Thailand (certificate of approval No. 128/2008). Written informed consent was obtained from all participants prior to conducting interviews and collecting blood samples.

\section{Studied population}

We performed a case-control study involving a sample population of 91 lung cancer patients and 84 control subjects. All individuals were residents of the northern region of Thailand and were of Thai ethnicity.

Study cases were recruited from patients who had been diagnosed with non-small cell lung cancer, i.e. adenocarcinoma, squamous cell carcinoma, large cell adenocarcinoma, and adenocarcinoma in situ, based on pathological and/or cytological reports. Neither chemotherapy nor irradiation had been administered to the participants within six months prior to the date of blood sample collection. The controls were healthy volunteers who had no prior history of any form of cancer. They were recruited as the spouses of lung cancer patients or non-blood-related volunteers who underwent routine annual health check-ups or as those who came along with the cancer patients at the time of their visit to the doctor.

All subjects were interviewed, using questionnaires, to obtain personal information including details of occupation, any history of lung disease, any history of invasive cancers among their firstdegree relatives, their personal habits (i.e. tobacco smoking, alcohol consumption, fermented tea leaf or betel nut chewing), and any environmental ex- 
posure to known lung carcinogens at their places of residence (i.e. home layout, location of kitchen, burning activities). 'Ever smokers', defined as persons having smoked daily for a year or longer, and 'Never smokers' defined as persons never having smoked regularly for a year or longer.

Peripheral blood samples of each participant were collected by venipuncture at the median cubital vein using a $6 \mathrm{ml}$ vacutainer coated with anticoagulant K2EDTA. All blood samples were processed to obtain cell lysates within $48 \mathrm{~h}$ after being collected.

\section{DNA extraction and SNP genotyping}

Total genomic DNA from peripheral blood leucocytes was extracted according to the standard inorganic salting out protocol [20]. Genotypes of TP53 Pro72Arg (rs1042522) and CDKN1A Ser31Arg (rs1801270) were investigated by polymerase chain reaction (PCR) with confronting two-pair primers (PCR-CTPP), as has been described by Hishida et al [21]. Genotypes of five SNPs, including MDM2 T309G (rs2279744), FAS G-1378A (rs2234767), FASLG C-844T (rs763110), TGFB1 T-1347C (rs1800469), and TGFB1 Pro10Leu (rs1800470), were detected by tetra-primer amplification refractory mutation system (T-ARMS-PCR), as has been previously described $[15,22,23]$. Detailed information of the studied SNPs are described in Table 1.

A new set of T-ARMS primers was designed and used for the genotyping of CCND1 G723A (rs9344) in this study, using the online web service primer design [24]. The T-ARMS primer sequences that were used are listed as follows: forward outer primer, $5^{\prime}$ AGT TCA TTT CCA ATC CGC CCT CCATG $3^{\prime}$; reverse inner primer, 5' CTG CCT GGG ACA TCA CCC TCA CTT CCT $3^{\prime}$; forward inner primer, $5^{\prime}$ TCC TCT CCA GAG TGA TCA AGT GTG ACACG $3^{\prime}$; reverse outer primer, $5^{\prime}$ GTT CTA GGA GCA GTG GAA GAA GCC GGTG $3^{\prime}$. Mismatches between the second bases from $3^{\prime}$ terminal of the inner primers and DNA templates were intentionally designed to further enhance specificity. The estimated sizes of three possible amplicons were 214 bp for CCND1 723G allele 169 bp for CCND1 723A allele and $317 \mathrm{bp}$ for the control products of two outer primers.

The primers and PCR cycling conditions for each SNP genotyping were modified from the relevant manufacturers' protocols according to the specified primers and the size of the amplicons needed to achieve distinguishable allelic-specific products (Table S1). Standard PCR conditions were estab- lished and initiated with an activation step $\left(95^{\circ} \mathrm{C}\right.$ for $12 \mathrm{~min}$ ), followed by amplification steps (30-40 cycles of $94^{\circ} \mathrm{C}$ for $20 \mathrm{~s}, 60-65^{\circ} \mathrm{C}$ for $40 \mathrm{~s}, 72^{\circ} \mathrm{C}$ for 20-60 s), and completed with a final extension step ( $72{ }^{\circ} \mathrm{C}$ for $7 \mathrm{~min}$ ) (Table S1).

The amplified products were subjected to electrophoresis in agarose gel. The percentage of agarose gel was depended on the size differences among the amplicons (Table S1). After electrophoresis, gels were stained with ethidium bromide and examined under Bio-Rad Gel Doc ${ }^{\mathrm{TM}} 2000$ Gel Documentation Systems using Quality One ${ }^{\circledR}$ program (Hercules, CA, USA). Amplified products were compared with 100 bp DNA GeneRuler (Thermo Scientific, Ontario, Canada), and a genotype of each sample was identified by comparing with three positive controls that had been genotyped previously by direct sequencing.

\section{Statistical analysis}

All statistical analyses were performed using IBM SPSS Statistics version 27.0 (IBM Corp., NY, USA) following the specific instructions of the statistical methods established for health care research. Continuous variables were transformed into categorical variables using appropriate cut-offs based on the acquired data. For descriptive data of the two subject groups, chi-square tests were used to test for the presence of any statistical differences at a significance level of $0.05(\alpha=0.05)$.

Any deviations from the Hardy-Weinberg equilibrium (HWE) of each SNP locus were assessed using the chi-square goodness-of-fit test statistics. Odds ratios (OR) and a 95\% confidence interval (CI) were used to evaluate the association between the risk of acquiring lung cancer and the influenced impact of an individual SNP or the combined effects of two SNPs within the same gene group, or the combined effects of the SNPs and any potential environmental risk factor. Stratified analysis for each environmental risk factor was done to determine any lung cancer risk among subjects that were more likely exposed to environmental lung carcinogens (by having kitchens inside their homes, being exposed to burning activities and those living in wooden houses). Using multivariable logistic regression, ORs were adjusted for age, gender and smoking status in the overall analyses of individual SNP genotypes and combinations of two SNPs within the same gene group; while ORs were adjusted for the smoking habits of the participants during the course of the subgroup analyses involving any of the environmental risk factors. 
Table 1 General information of the studied SNPs.

\begin{tabular}{|c|c|c|c|c|c|}
\hline Abbrev. $^{\mathrm{a}}$ & Gene name $^{\mathrm{a}}$ & dbSNP ID & HGVS nomenclature ${ }^{\mathrm{b}}$ & $\begin{array}{l}\text { Common name } \\
\text { (Alternate name) }\end{array}$ & $\mathrm{GMAF}^{\mathrm{c}}$ \\
\hline \multicolumn{6}{|c|}{ Cell cycle control genes } \\
\hline TP53 & Tumor protein p53 & rs1042522 & NM_000546.6:c.215C>G (p.Pro72Arg) & Pro72Arg (Arg72Pro) & $0.45707(\mathrm{G})$ \\
\hline MDM2 & MDM2 proto-oncogene & rs2279744 & $\mathrm{NM}_{-}^{-}$002392.5:c.14+309T>G & T309G (SNP309) & $0.36661(\mathrm{G})$ \\
\hline CDKN1A & $\begin{array}{l}\text { Cyclin dependent kinase } \\
\text { inhibitor } 1 \mathrm{~A}\end{array}$ & rs 1801270 & $\mathrm{NM}_{-}^{-}$000389.5:c.93C >A (p.Ser31Arg) & Ser31Arg & $0.25619(\mathrm{~A})$ \\
\hline CCND1 & Cyclin D1 & rs9344 & NM_053056.2:c.723G >A (p.Pro241=) & G723A (G870A) & $0.41354(\mathrm{~A})$ \\
\hline \multicolumn{6}{|c|}{ Apoptosis genes } \\
\hline FASLG & Fas ligand & rs763110 & NM_000639.2:c.-844C>T & C-844T (T-844C) & $0.46965(\mathrm{C})$ \\
\hline FAS & Fas cell surface death receptor & rs2234767 & $\mathrm{NM}_{-}^{-}$000043.5:c.-1378G $>\mathrm{A}$ & G-1378A (G-1377A) & $0.18411(\mathrm{~A})$ \\
\hline \multicolumn{6}{|c|}{ Inflammation cytokine genes } \\
\hline \multirow[t]{2}{*}{ TGFB1 } & $\begin{array}{l}\text { Transforming growth factor } \\
\text { beta } 1\end{array}$ & rs1800469 & NM_000660.6:c.-1347T>C & T-1347C (C-509T) & $0.36801(\mathrm{~T})$ \\
\hline & & rs1800470 & NM_000660.7:c.29C>T (p.Pro10Leu) & Pro10Leu (Leu10Pro) & $0.45467(\mathrm{C})$ \\
\hline
\end{tabular}

${ }^{a}$ According to the HUGO Gene Nomenclature Committee at the European Bioinformatics Institute.

${ }^{b}$ According to the recommendations of the Human Genome Variation Society (HGVS).

${ }^{\mathrm{c}}$ Global minor allele frequency (GMAF) in the ClinVar database.

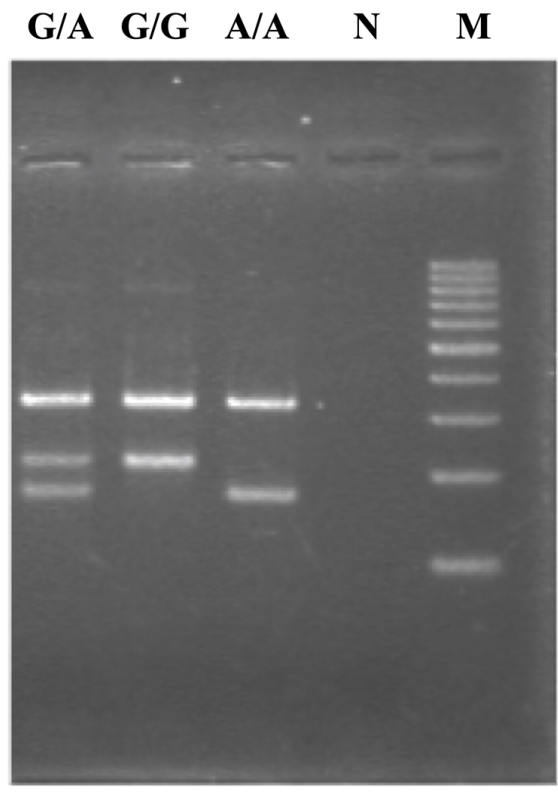

$400 \mathrm{bp}$ 300 bp 200 bp

$100 \mathrm{bp}$

Fig. 1 CCND1 G723A genotyping by T-ARMS-PCR technique. Three possible genotype products are as follows; G/A (214-bp and 169-bp), G/G (214-bp) and A/A (169bp). The internal control products are 317-bp amplicons. $\mathrm{N}$ : negative control, M: 100-bp DNA ladder.

\section{RESULTS}

The demographic characteristics of the patients and the controls, together with their prevalence of exposure to the listed environmental lung cancer risk factors, are shown in Table 2 . The mean age of the patients with ever smoking habits were higher than the healthy control group. Among the environmen- tal factors, significant differences were observed between cancer patients and the control groups only in proportion to their smoking status $(\mathrm{P}<0.01)$.

Individual SNP genotyping was successfully done with either PCR-CTPP or T-ARMS-PCR. The establishment of newly designed T-ARMS-PCR primers for the genotyping of CCND1 G723A is shown in Fig. 1. Using the gel electrophoresis technique, one of three possible genotypes of CCND1 G723A polymorphism, including G/A (214-bp and 169-bp), G/G (214-bp) and A/A (169-bp), was identified for each sample. SNP genotyping of the randomly selected samples was twice repeated meticulously, and all genotyping results were found to be consistent.

Using the chi-square goodness-of-fit test, we found that the genotype distributions of all 8 selected SNP loci agreed with those of the HWE $(P>0.05)$ in both patients and controls. We then used a dominant comparison model by combining heterozygous and homozygous variants and using the homozygous wild type as a reference group. Multivariate logistic regression analyses (adjusted for age, gender and smoking status) revealed that no significant effects of any variant alleles were observed on the ORs of lung cancer risk at each SNP (Table 3).

We next examined the combined effects of two SNPs within each group of genes regulating the pathway/process on lung cancer risk. According to the ORs and 95\% CIs (Table 4), individuals with both -1347T and 10Pro alleles of TGFB1 had significantly increased their risk of developing lung cancer $(\mathrm{OR}=2.90,95 \% \mathrm{CI}=1.01-8.4, \mathrm{P}=0.049$ ) in comparison with those that did not carry these two alleles. No significant association was found 
Table 2 Characteristics of the studied population.

\begin{tabular}{|c|c|c|c|}
\hline Variable & Control group, n(\%) & Patient group, $\mathrm{n}(\%)$ & $\mathrm{P}$ value \\
\hline Sample size & $\mathrm{N}=84$ & $\mathrm{~N}=91$ & \\
\hline \multicolumn{4}{|l|}{ Age } \\
\hline Mean \pm SD & $52.5 \pm 9.11$ & $62.2 \pm 9.37$ & $<0.001$ \\
\hline (Minimum-Maximum) & $26-77$ & $40-84$ & \\
\hline Gender & & & 0.22 \\
\hline Female & $41(48.8)$ & $36(38.7)$ & \\
\hline Male & $43(51.2)$ & $55(61.3)$ & \\
\hline \multicolumn{4}{|l|}{ Histological type of lung cancer } \\
\hline Adenocarcinoma (ADC) & & $58(63.7)$ & \\
\hline Squamous cell carcinoma (SQC) & & $25(27.5)$ & \\
\hline Large cell carcinoma (LAC) & & $5(5.5)$ & \\
\hline Adenocarcinoma in situ (AIS) & & $3(3.3)$ & \\
\hline Smoking status & & & $<0.001$ \\
\hline Never & $49(58.3)$ & $16(17.6)$ & \\
\hline Ever & $35(41.7)$ & $75(82.4)$ & \\
\hline Kitchen location & & & 0.63 \\
\hline Outside house & $44(52.4)$ & $51(56.0)$ & \\
\hline Inside house & $40(47.6)$ & $40(44.0)$ & \\
\hline Housing materials & & & 0.79 \\
\hline Concrete house & $33(39.3)$ & $34(37.4)$ & \\
\hline Wooden house & $51(60.7)$ & $57(62.6)$ & \\
\hline Burning activity & & & 0.48 \\
\hline Once a week or none & $31(36.9)$ & 29 (31.9) & \\
\hline Twice a week or more & $53(63.1)$ & $62(68.1)$ & \\
\hline
\end{tabular}

Table 3 Effects of individual SNPs on the risk of being diagnosed with lung cancer. Odds ratio (OR) and 95\% confidence intervals (CI) were estimated using multivariate logistic regression model and were adjusted for age, gender and smoking status.

\begin{tabular}{|c|c|c|c|c|c|}
\hline SNPs & Controls, $\mathrm{n}(\%)$ & Patients, n(\%) & OR & $(95 \% \mathrm{CI})$ & $P$ value \\
\hline \multicolumn{6}{|c|}{ Cell cycle control genes } \\
\hline TP53 Pro72Arg & 83 & 91 & & & \\
\hline Arg/Arg & $19(22.9)$ & $23(25.3)$ & 1.00 & (reference) & \\
\hline Arg/Pro + Pro/Pro & $64(77.1)$ & $68(74.7)$ & 0.86 & $(0.376-1.95)$ & 0.71 \\
\hline$M D M 2 \mathrm{~T} 309 \mathrm{G}$ & 83 & 91 & & & \\
\hline $\mathrm{T} / \mathrm{T}$ & $16(19.3)$ & $20(22.0)$ & 1.00 & (reference) & \\
\hline $\mathrm{T} / \mathrm{G}+\mathrm{G} / \mathrm{G}$ & 67 (80.7) & $71(78.0)$ & 0.75 & $(0.307-1.82)$ & 0.52 \\
\hline CDKN1A Ser31Arg & 83 & 91 & & & \\
\hline Ser/Ser & $22(26.5)$ & $28(30.8)$ & 1.00 & (reference) & \\
\hline Ser/Arg + Arg/Arg & $61(73.5)$ & $63(69.2)$ & 1.23 & $(0.56-2.72)$ & 0.61 \\
\hline CCND1 G723A & 80 & 90 & & & \\
\hline $\mathrm{G} / \mathrm{G}$ & $7(8.8)$ & $14(15.6)$ & 1.00 & (reference) & \\
\hline $\mathrm{G} / \mathrm{A}+\mathrm{A} / \mathrm{A}$ & $73(91.2)$ & $76(84.4)$ & 0.44 & $(0.138-1.43)$ & 0.17 \\
\hline \multicolumn{6}{|l|}{ Apoptosis genes } \\
\hline FASLG C-844T & 81 & 90 & & & \\
\hline $\mathrm{T} / \mathrm{T}$ & $11(13.6)$ & $9(10.0)$ & 1.00 & (reference) & \\
\hline $\mathrm{T} / \mathrm{C}+\mathrm{C} / \mathrm{C}$ & $70(86.4)$ & $81(90.0)$ & 0.95 & $(0.320-2.79)$ & 0.92 \\
\hline FAS G-1378A & 79 & 90 & & & \\
\hline $\mathrm{G} / \mathrm{G}$ & $24(30.4)$ & $31(34.4)$ & 1.00 & (reference) & \\
\hline $\mathrm{G} / \mathrm{A}+\mathrm{A} / \mathrm{A}$ & $55(69.6)$ & 59 (65.6) & 0.93 & $(0.43-2.04)$ & 0.86 \\
\hline \multicolumn{6}{|c|}{ Inflammatory cytokine genes } \\
\hline TGFB1 T-1347C & 73 & 88 & & & \\
\hline $\mathrm{C} / \mathrm{C}$ & $18(24.7)$ & $9(10.2)$ & 1.00 & (reference) & \\
\hline $\mathrm{C} / \mathrm{T}+\mathrm{T} / \mathrm{T}$ & $55(75.3)$ & $79(89.8)$ & 2.57 & $(0.90-7.4)$ & 0.079 \\
\hline TGFB1 Pro10Leu & 80 & 90 & & & \\
\hline Leu/Leu & $12(15.0)$ & $7(7.8)$ & 1.00 & (reference) & \\
\hline Leu/Pro + Pro/Pro & $68(85.0)$ & $83(92.2)$ & 2.13 & $(0.67-6.8)$ & 0.20 \\
\hline
\end{tabular}


Table 4 Combined effects of two SNPs on the risk of being diagnosed with lung cancer. Odds ratio (OR) and 95\% confidence intervals (CI) were estimated using multivariate logistic regression model and were adjusted for age, gender and smoking status.

\begin{tabular}{|c|c|c|c|c|c|}
\hline SNPs & Controls, $\mathrm{n}(\%)$ & Patients, n(\%) & OR & $(95 \% \mathrm{CI})$ & $P$ value \\
\hline \multicolumn{6}{|l|}{ Cell cycle control genes } \\
\hline The others & $32(38.6)$ & $37(40.7)$ & 1.00 & (reference) & \\
\hline $\begin{array}{l}\text { Pro/- \& G/- } \\
\text { Ser31Arro }\end{array}$ & $51(61.4)$ & $54(59.3)$ & 0.83 & $(0.398-1.71)$ & \multirow[t]{2}{*}{0.61} \\
\hline $\begin{array}{l}\text { Ser31Arg } \\
\text { The others }\end{array}$ & $\begin{array}{c}83 \\
36(43.4)\end{array}$ & $41 \stackrel{91}{(45.1)}$ & 1.00 & (reference) & \\
\hline G/- \& Arg/- & $47(56.6)$ & $50(54.9)$ & 1.18 & $(0.57-2.43)$ & \multirow[t]{2}{*}{0.66} \\
\hline TP53 Pro72Arg \& CCND1 G723A & 80 & 90 & & & \\
\hline The others & $20(25.0)$ & $33(36.7)$ & 1.00 & (reference) & \multirow[b]{2}{*}{0.098} \\
\hline $\begin{array}{l}\text { Pro/- \& A/- } \\
\text { MDM2 T309G \& CDKN1A Ser31Arg }\end{array}$ & $60(75.0)$ & $57(63.3)$ & 0.51 & $(0.228-1.13)$ & \\
\hline The others & $30(36.1)$ & $42(46.2)$ & 1.00 & (reference) & \multirow{3}{*}{0.49} \\
\hline G/- \& Arg/- & $53(63.9)$ & $49(53.8)$ & 0.78 & $(0.375-1.61)$ & \\
\hline MDM2 T309G \& CCND1 G723A & 80 & 90 & & & \\
\hline The others & $22(27.5)$ & $32(35.6)$ & 1.00 & (reference) & \multirow{3}{*}{0.10} \\
\hline G/- \& A/- & $58(72.5)$ & $58(64.4)$ & 0.51 & $(0.230-1.14)$ & \\
\hline CDKN1A Ser31Arg \& CCND1 G723A & 80 & 90 & & & \\
\hline The others & $26(32.5)$ & $39(43.3)$ & 1.00 & (reference) & \multirow[b]{2}{*}{0.38} \\
\hline Arg/- \& A/- & $54(67.5)$ & $51(56.7)$ & 0.72 & $(0.341-1.51)$ & \\
\hline \multicolumn{6}{|l|}{ Apoptosis genes } \\
\hline FASLG C-844T \& FAS G-1378A & 79 & 90 & & \multirow{2}{*}{\multicolumn{2}{|c|}{ (reference) }} \\
\hline The others & $33(41.8)$ & $34(37.8)$ & 1.00 & & \\
\hline $\mathrm{C} /-$ \& A/- & $46(58.2)$ & $56(62.2)$ & 1.10 & $(0.53-2.32)$ & 0.80 \\
\hline \multicolumn{6}{|l|}{ Inflammatory cytokine genes } \\
\hline TGFB1 T-1347C \& TGFB1 Pro10Leu & 72 & 88 & & \multirow{2}{*}{\multicolumn{2}{|c|}{ (reference) }} \\
\hline The others & $18(25.0)$ & $9(10.2)$ & 1.00 & & \\
\hline T/- + Pro/- & $54(75.0)$ & $79(89.8)$ & 2.90 & $(1.01-8.4)$ & $0.049 *$ \\
\hline
\end{tabular}

$* \mathrm{P}<0.05$.

Table 5 Effects of individual SNPs on the risk of being diagnosed with lung cancer among individuals having kitchens inside their homes. Odds ratio (OR) and 95\% confidence intervals (CI) were estimated using multivariate logistic regression model and were adjusted for age, gender and smoking status.

\begin{tabular}{|c|c|c|c|c|c|}
\hline SNPs & Controls, n(\%) & Patients, n(\%) & OR & $(95 \% \mathrm{CI})$ & $\mathrm{P}$ value \\
\hline \multicolumn{6}{|c|}{ Cell cycle control genes } \\
\hline TP53 Pro72Arg & 40 & 40 & & & \\
\hline Arg/Arg & $10(25.0)$ & $12(30.0)$ & 1.00 & (reference) & \\
\hline Arg/Pro + Pro/Pro & $30(75.0)$ & $28(70.0)$ & 0.90 & $(0.285-2.85)$ & 0.86 \\
\hline MDM2 T309G & 40 & 40 & & & \\
\hline $\mathrm{T} / \mathrm{T}$ & $11(27.5)$ & $9(22.5)$ & 1.00 & (reference) & \\
\hline $\mathrm{T} / \mathrm{G}+\mathrm{G} / \mathrm{G}$ & $29(72.5)$ & $31(77.5)$ & 1.25 & $(0.380-4.1)$ & 0.71 \\
\hline CDKN1A Ser31Arg & 40 & 40 & & & \\
\hline Ser/Ser & $9(22.5)$ & $10(25.0)$ & 1.00 & (reference) & \\
\hline Ser/Arg + Arg/Arg & $31(77.5)$ & $30(75.0)$ & 1.03 & $(0.311-3.40)$ & 0.96 \\
\hline CCND1 G723A & 39 & 39 & & & \\
\hline $\mathrm{G} / \mathrm{G}$ & $2(5.1)$ & 7 (17.9) & 1.00 & (reference) & \\
\hline $\mathrm{G} / \mathrm{A}+\mathrm{A} / \mathrm{A}$ & 37 (94.9) & $32(82.1)$ & 0.152 & $(0.023-1.03)$ & 0.053 \\
\hline \multicolumn{6}{|l|}{ Apoptosis genes } \\
\hline FASLG C-844T & 38 & 39 & & & \\
\hline $\mathrm{T} / \mathrm{T}$ & $7(18.4)$ & $4(10.3)$ & 1.00 & (reference) & \\
\hline $\mathrm{T} / \mathrm{C}+\mathrm{C} / \mathrm{C}$ & $31(81.6)$ & 35 (89.7) & 1.08 & $(0.250-4.7)$ & 0.91 \\
\hline FAS G-1378A & 38 & 39 & & & \\
\hline $\mathrm{G} / \mathrm{G}$ & $13(34.2)$ & $11(28.2)$ & 1.00 & (reference) & \\
\hline $\mathrm{G} / \mathrm{A}+\mathrm{A} / \mathrm{A}$ & $25(65.8)$ & $28(71.8)$ & 1.52 & $(0.49-4.7)$ & 0.47 \\
\hline \multicolumn{6}{|c|}{ Inflammatory cytokine genes } \\
\hline TGFB1 T-1347C & 32 & 38 & & & \\
\hline $\mathrm{C} / \mathrm{C}$ & $11(34.4)$ & $4(10.5)$ & 1.00 & (reference) & \\
\hline $\mathrm{C} / \mathrm{T}+\mathrm{T} / \mathrm{T}$ & $21(65.6)$ & $34(89.5)$ & 5.9 & $(1.32-26.5)$ & $0.02 *$ \\
\hline TGFB1 Pro10Leu & 37 & 39 & & & \\
\hline Leu/Leu & $7(18.9)$ & $4(10.3)$ & 1.00 & (reference) & \\
\hline Leu/Pro + Pro/Pro & $30(81.1)$ & $35(89.7)$ & 2.43 & $(0.54-10.9)$ & 0.25 \\
\hline
\end{tabular}

$* \mathrm{P}<0.05$. 
Table 6 Effects of individual SNPs on the risk of being diagnosed with lung cancer among individuals living in wooden houses. Odds ratio (OR) and 95\% confidence intervals (CI) were estimated using multivariate logistic regression model and were adjusted for age, gender and smoking status.

\begin{tabular}{|c|c|c|c|c|c|}
\hline SNPs & Controls, $\mathrm{n}(\%)$ & Patients, $\mathrm{n}(\%)$ & OR & $(95 \% \mathrm{CI})$ & $P$ value \\
\hline \multicolumn{6}{|c|}{ Cell cycle control genes } \\
\hline Arg/Arg & $9(18.0)$ & $12(21.1)$ & 1.00 & (reference) & \multirow{3}{*}{0.80} \\
\hline Arg/Pro + Pro/Pro & $41(82.0)$ & $45(78.9)$ & 1.18 & $(0.338-4.1)$ & \\
\hline MDM2 T309G & 50 & 57 & & & \\
\hline $\mathrm{T} / \mathrm{T}$ & $9(18.0)$ & $13(22.8)$ & 1.00 & (reference) & \multirow{3}{*}{0.47} \\
\hline $\mathrm{T} / \mathrm{G}+\mathrm{G} / \mathrm{G}$ & $41(82.0)$ & $44(77.2)$ & 0.64 & $(0.189-2.15)$ & \\
\hline CDKN1A Ser31Arg & 50 & 57 & & & \\
\hline Ser/Ser & $10(20.0)$ & $21(36.8)$ & 1.00 & (reference) & \multirow{3}{*}{0.61} \\
\hline Ser/Arg + Arg/Arg & $40(80.0)$ & $36(63.2)$ & 0.75 & $(0.247-2.26)$ & \\
\hline CCND1 G723A & 48 & 57 & & & \\
\hline G/G & $5(10.4)$ & $3(5.3)$ & 1.00 & (reference) & \multirow[b]{2}{*}{0.27} \\
\hline $\mathrm{G} / \mathrm{A}+\mathrm{A} / \mathrm{A}$ & $43(89.6)$ & $54(94.7)$ & 3.13 & $(0.41-23.7)$ & \\
\hline \multicolumn{6}{|l|}{ Apoptosis genes } \\
\hline $\begin{array}{l}\text { FASLG C-844T } \\
\mathrm{T} / \mathrm{T}\end{array}$ & $\begin{array}{c}49 \\
10(20.4)\end{array}$ & $\begin{array}{c}47 \\
7(12.3)\end{array}$ & & (reference) & \multirow{3}{*}{0.65} \\
\hline $\begin{array}{l}\mathrm{T} / \mathrm{T} \\
\mathrm{T} / \mathrm{C}+\mathrm{C} / \mathrm{C}\end{array}$ & $\begin{array}{l}10(20.4) \\
39(79.6)\end{array}$ & $\begin{array}{c}7(12.3) \\
40(87.7)\end{array}$ & $\begin{array}{l}1.00 \\
1.37\end{array}$ & $\begin{array}{l}\text { (reference) } \\
(0.353-5.3)\end{array}$ & \\
\hline FAS G-1378A & 48 & 57 & & & \\
\hline $\mathrm{G} / \mathrm{G}$ & $15(31.2)$ & $20(35.1)$ & 1.00 & (reference) & \multirow[b]{2}{*}{0.69} \\
\hline $\mathrm{G} / \mathrm{A}+\mathrm{A} / \mathrm{A}$ & $33(68.8)$ & $37(64.9)$ & 1.24 & $(0.43-3.58)$ & \\
\hline \multicolumn{6}{|c|}{ Inflammatory cytokine genes } \\
\hline TGFB1 T-1347C & 43 & 56 & & & \multirow{4}{*}{0.058} \\
\hline $\mathrm{C} / \mathrm{C}$ & $12(27.9)$ & 5 (8.9) & 1.00 & (reference) & \\
\hline $\mathrm{C} / \mathrm{T}+\mathrm{T} / \mathrm{T}$ & $31(72.1)$ & 51 (91.1) & 4.2 & $(0.95-18.5)$ & \\
\hline TGFB1 Pro10Leu & 49 & 57 & & & \\
\hline Leu/Leu & $7(14.3)$ & $4(7.0)$ & 1.00 & (reference) & \\
\hline Leu/Pro + Pro/Pro & $42(85.7)$ & $53(93.0)$ & 4.1 & $(0.77-21.6)$ & 0.099 \\
\hline
\end{tabular}

Table 7 Effects of individual SNPs on the risk of being diagnosed with lung cancer among individuals exposed to burning activities. Odds ratio (OR) and 95\% confidence intervals (CI) were estimated using multivariate logistic regression model and were adjusted for age, gender and smoking status.

\begin{tabular}{|c|c|c|c|c|c|}
\hline SNPs & Controls, n(\%) & Patients, n(\%) & OR & $(95 \% \mathrm{CI})$ & $P$ value \\
\hline \multicolumn{6}{|c|}{ Cell cycle control genes } \\
\hline $\begin{array}{l}\text { TP53 Pro72Arg } \\
\text { Arg/Arg }\end{array}$ & $\begin{array}{c}53 \\
9(17.0)\end{array}$ & $\begin{array}{c}62 \\
12(19.4)\end{array}$ & 1.00 & (reference) & \multirow{3}{*}{0.36} \\
\hline Arg/Pro + Pro/Pro & $44(83.0)$ & $50(80.6)$ & 0.58 & (0.180-1.87) & \\
\hline MDM2 T309G & 53 & 62 & & & \\
\hline $\mathrm{T} / \mathrm{T}$ & $11(20.8)$ & $16(25.8)$ & 1.00 & (reference) & \multirow[b]{2}{*}{0.32} \\
\hline $\begin{array}{l}\mathrm{T} / \mathrm{G}+\mathrm{G} / \mathrm{G} \\
C D K N 1 \mathrm{~A} \text { Ser31Aro }\end{array}$ & $42(79.2)$ & $\begin{array}{l}46(74.2) \\
62\end{array}$ & 0.58 & $(0.195-1.71)$ & \\
\hline $\begin{array}{l}\text { CDKN1A Ser31Arg } \\
\text { Ser/Ser }\end{array}$ & $16(30.2)$ & $16(25.8)$ & 1.00 & (reference) & \multirow{3}{*}{0.21} \\
\hline Ser/Arg + Arg/Arg & $37(69.8)$ & $46(74.2)$ & 1.94 & $(0.70-5.4)$ & \\
\hline CCND1 G723A & 51 & 61 & & & \\
\hline G/G & $5(9.8)$ & $9(14.8)$ & 1.00 & (reference) & \multirow[b]{2}{*}{0.28} \\
\hline $\mathrm{G} / \mathrm{A}+\mathrm{A} / \mathrm{A}$ & $46(90.2)$ & $52(85.2)$ & 0.44 & $(0.100-1.96)$ & \\
\hline \multicolumn{6}{|l|}{ Apoptosis genes } \\
\hline FASLG C-844T & 51 & 61 & & & \multirow{4}{*}{0.89} \\
\hline $\mathrm{T} / \mathrm{T}$ & 7 (13.7) & $5(8.2)$ & 1.00 & (reference) & \\
\hline $\mathrm{T} / \mathrm{C}+\mathrm{C} / \mathrm{C}$ & $44(86.3)$ & $56(91.8)$ & 1.11 & $(0.266-4.6)$ & \\
\hline FAS G-1378A & 51 & 61 & & & \\
\hline $\mathrm{G} / \mathrm{G}$ & $16(31.4)$ & $20(32.8)$ & 1.00 & (reference) & \multirow[b]{2}{*}{0.89} \\
\hline $\mathrm{G} / \mathrm{A}+\mathrm{A} / \mathrm{A}$ & $35(68.6)$ & $41(67.2)$ & 1.07 & $(0.390-2.95)$ & \\
\hline \multicolumn{6}{|c|}{ Inflammatory cytokine genes } \\
\hline TGFB1 T-1347C & 46 & 59 & & & \multirow{5}{*}{0.63} \\
\hline $\mathrm{C} / \mathrm{C}$ & $11(23.9)$ & $9(15.3)$ & 1.00 & (reference) & \\
\hline $\mathrm{C} / \mathrm{T}+\mathrm{T} / \mathrm{T}$ & $35(76.1)$ & $50(84.7)$ & 1.35 & $(0.397-4.6)$ & \\
\hline TGFB1 Pro10Leu & 51 & 61 & & & \\
\hline Leu/Leu & $8(15.7)$ & 7 (11.5) & 1.00 & (reference) & \\
\hline Leu/Pro + Pro/Pro & $43(84.3)$ & $54(88.5)$ & 1.44 & $(0.384-5.4)$ & 0.59 \\
\hline
\end{tabular}


between any other combination of two SNPs within the same gene groups and the risk of acquiring lung cancer.

Lung cancer susceptibility risks were evaluated among individuals who were exposed to each of the three environmental risk factors, including those having kitchens inside their houses (Table 5), those living in wooden houses (Table 6), and those exposed to burning activities in and around their homes (Table 7). Interestingly, a significant association was observed between a combined effect of SNPs and the location of the kitchen for lung cancer risk. Among participants who had kitchens inside their houses, TGFB1-1347T allele carriers were associated with a significant increase risk of lung cancer $(\mathrm{OR}=5.9,95 \% \mathrm{CI}=1.32-26.5, \mathrm{P}=0.020$, adjusted for age, gender and smoking status) in comparison with the TGFB1-1347 C/C genotype carriers. Albeit somewhat non-significant, two SNPs in the TGFB1 gene revealed potential associations with an elevated risk for developing lung cancer among those participants residing in wooden houses $(\mathrm{P}<0.10)$ (Table 6). However, there was no significant association observed between SNP and lung cancer risk among those exposed to burning activities around their homes (Table 7).

\section{DISCUSSION}

In previous studies, the variant alleles of all eight studied SNPs in this study had been reported to affect changes in either protein functions or gene expression and were found to be significantly associated with lung cancer risk. However, effects of these SNP variations and the potential environmental risk factors for susceptibility to lung cancer among the people of northern Thailand has not yet been evaluated. Although, there were no significant associations between each of the studied SNPs and a risk for lung cancer in the multivariate analyses, a potential association between the TGFB1 T-1347C polymorphism and a risk of acquiring lung cancer was observed. It was likely that individuals carrying -1347T alleles would have a 2-fold increased risk of developing lung cancer (Table 3 ). In a stratified analysis of smoking history, Park et al [16] found that having at least one $-1347 \mathrm{~T}$ allele was associated with a 3.8-fold increased risk of acquiring lung cancer among heavy smokers ( $\geqslant 30$ pack-years). Moreover, a 1.2-fold increased risk of acquiring lung cancer was found among Asian individuals who carried at least one TGFB1-1347T allele in the metaanalysis [25]. However, a significant inverse association was observed between the TGFB1 T-1347C polymorphism and a lung cancer risk in several previous studies conducted among Asian populations. Kang et al [25] found that the TGFB1 -1347T allele carriers displayed $27 \%$ and $37 \%$ reduced risks of acquiring lung cancer and lung adenocarcinoma, respectively. These results were obtained from multivariate analyses after being adjusted for any potential confounding factors, including age, gender, smoking status, and, especially, pack-years of smoking [26].

Although we observed a significant association between a combination of the two SNPs in the TGFB1 gene, namely -1347T and 10Pro alleles, these two SNPs were found to be in strong linkage disequilibrium $[17,18]$. Both TGFB1 T-1347C and Pro10Leu are known to be functional polymorphisms that affect the levels of TGFB1 transcription and TGF- $\beta 1$ secretion [27]. However, there has been no experimental evidence showing the effect of combining TGFB1 T-1347C and Pro10Leu polymorphisms on the levels of TGF- $\beta 1$ [28]. Based on the functional studies of each SNP, it has been hypothesized that synergy between TGFB1 upregulation (caused by -1347T allele) together with high levels of TGF- $\beta 1$ secretions (caused by 10Pro allele) might lead to a substantial increase in the plasma levels of TGF- $\beta 1$. Together with an inactivation of key mediators in TGF- $\beta 1$ signaling (e.g. SMAD4), the role of TGF- $\beta 1$ was switched from acting as a tumor-suppressive, by inducing cell cycle arrest and apoptosis, to serving as a tumor-promoting factor via immunosuppression by highly secreted TGF- $\beta 1$ proteins in the tumor microenvironment $[29,30]$. However, our observations of significant increases in the risk of developing lung cancer among individuals who carry both $-1347 \mathrm{~T}$ and 10Pro alleles of TGFB1 were inconsistent with those of a previous study conducted upon the Korean population [26]. Kang et al [26] found that the TGFB1 haplotype, with $-1347 \mathrm{~T}$ and 10Pro, was significantly associated with a $25 \%$ reduction in the risk of being diagnosed with lung adenocarcinoma. However, neither overall lung cancer nor squamous cell carcinoma subtypes were related to the TGFB1 haplotype with -1347C and 10Leu after adjustments were made for age, gender, smoking status, and pack-years of smoking.

We have observed a significant association between the TGFB1-1347T allele and a risk of developing lung cancer among individuals who had kitchens inside their houses. TGFB1-1347T allele carriers were associated with about a 5-fold increased risk of being diagnosed with lung cancer in comparison 
with the TGFB1-1347C allele carriers. Having a kitchen inside the house was found to have significantly contributed to a high prevalence (45.6\%) of TP53 somatic mutations that were observed in lung cancer patients in comparison with lung cancer patients who had kitchens outside their houses (16.7\%) [10]. The severity of this factor with regard to an increased risk of lung cancer may depend upon certain characteristics or features of the subject's home, such as the number of windows, the presence of a chimney, proper air ventilation, and any cooking activities that occur inside the kitchen that involve fuel used for cooking [31]. Moreover, the use of biomass fuels for cooking was recently found to be associated with long-term exposure to cancer-causing substances, including polycyclic aromatic hydrocarbons, such as benz[a]anthracene, benzo[k]fluoranthrene, benzo[a]pyrene, and their nitro derivatives, that were found to have accumulated in the homes of lung cancer patients residing in Chiang Mai Province [9]. Undeniably, inhalation of these substances contributes to the development of lung cancer. On the other hand, the longer one spends inside their home, the greater the risk they appear to have of developing lung cancer. Trans, trans-2,4-decadienal (tt-DDE or 2,4-De), a specific type of dienaldehyde in cooking oil fumes was found to be associated with increased cell proliferation and enhanced production of tumour necrosis factor- $\alpha$ (TNF- $\alpha$ ) in human bronchial epithelial cells [32]. This TNF- $\alpha$ in cooperation with TGF- $\beta 1$, which is up-regulated by the $-1347 \mathrm{~T}$ allele, could promote cancer progression through epithelial-mesenchymal transition (EMT) [29,33]. Thus, individuals who had kitchens inside their houses while carrying TGFB1-1347T alleles could have an increased risk of developing lung cancer.

At first, we expected that the participants who were exposed to burning activities around their homes would have a greater likelihood of long-term exposure to airborne particulate matters that would subsequently lead to lung cancer. Vegetative burning was the main source of particulate matters, with an aerodynamic diameter of smaller than $10 \mu \mathrm{m}$ (46-82\% of the total $\mathrm{PM}_{10}$ concentration), during the period of June 2005 to June 2006 in Chiang Mai and Lamphun Provinces [8]. Burning in open fields is a convenient way to get rid of agricultural residues after harvesting crops. Smoke from bush fires in this region and from surrounding countries during the dry season are brought here by southwesterly winds that also contribute to the accumulation of particulate matters in the air of Chiang Rai Province, the northern most province of Thailand $[34,35]$. For every $10-\mu \mathrm{g} / \mathrm{m}^{3}$ change in the exposure of $\mathrm{PM}_{10}$, the risk of developing lung cancer and lung adenocarcinoma would increase by $9 \%$ and $29 \%$, respectively [36]. However, we did not observe any significant association between each of the studied SNPs and the risk of being diagnosed with lung cancer among participants who were exposed to burning activities around their homes. However, having or not having exposure to burning activities around the home may depend upon a number of other factors, such as the location of the house (urban or rural area), the weather, the crop seasons, and the amount of agricultural waste or household waste that is being produced by members of that household. More detailed information about the exposure to burning activities may be useful in defining this parameter and in the application of the stratified analyses by a subgroup of the burning activities.

Although we have observed some association between the candidate SNPs in genes regulating pathways/processes and a risk of developing lung cancer in the population of northern Thailand, we were faced with some limitations during the course of conducting this research. The limitations were solely due to the small sample size. The limited number of samples in both patient and control groups affected the statistical power in the analyses of multivariate logistic regression, especially with regard to the stratified subject groups who were exposed to the designated environmental risk factors. Although the sample size of this study is acceptable for multivariate logistic regression analyses (at least ten samples per one independent variable) [37], it was still less than the suggested level (at least two controls per one case) [38], resulting in low statistical power. The expansion of the population size of the study by recruiting more lung cancer patients and even more unrelated control subjects from the same district may be beneficial for an incoming case-control study. With an adequate sample size, consideration of other risk factors for lung cancer, such as education level, occupation, exposure to second-hand smoke, previous experience with lung diseases, the use of household solid fuels, and a family history of lung cancer, as well as menstrual and reproductive factors in women, can be evaluated for any association with lung cancer development in further studies. 


\section{CONCLUSION}

In conclusion, we have observed significant associations between TGFB1-1347T allele and either TGFB1 10Pro allele or having kitchen inside the house with an elevated risk of non-small cell lung cancer among the population of northern Thailand. Our results suggest that there are mutual contributions of individual genetic susceptibility and exposure to environmental carcinogens for the development of lung cancer in this region.

\section{Appendix A. Supplementary data}

Supplementary data associated with this article can be found at http://dx.doi.org/10.2306/ scienceasia1513-1874.2020.079.

Acknowledgements: The authors wish to thank all of those who participated in this research project by providing necessary information through the interview forms and by donating blood samples for the SNP genotyping process. This research project was jointly funded by the Royal Golden Jubilee PhD Programme (grant no. PHD/0077/2550), the Thailand Science Research and Innovation and the National Research Council of Thailand. JK gratefully acknowledges the financial support provided by CMU Mid-Career Research Fellowship program, Chiang Mai University, Thailand.

\section{REFERENCES}

1. Bray F, Ferlay J, Soerjomataram I, Siegel RL, Torre LA, Jemal A (2018) Global cancer statistics 2018: GLOBOCAN estimates of incidence and mortality worldwide for 36 cancers in 185 countries. CA Cancer $J$ Clin 68, 394-424.

2. Sawanyawisuth K, Wongkham C, Pairojkul C, Wongkham S (2018) Translational cancer research towards Thailand 4.0. ScienceAsia 44S, 11-18.

3. Imsamran W, Pattatang A, Supattagorn P, Chiawiriyabunya I, Namthaisong K, Wongsena M, Puttawibul P, Chitapanarux I, et al (2018) Cancer in Thailand, vol. IX, 2013-2015, New Thammada Press, Bangkok, Thailand.

4. Chitapanarux I, Srisukho S (2014) Cancer Incidence and Mortality in Chiang Mai, 2011, Academic Publishing Unit, Faculty of Medicine, Chiang Mai University, Chiang Mai, Thailand.

5. Virani S, Bilheem S, Chansaard W, Chitapanarux I, Daoprasert K, Khuanchana S, Leklob A, Pongnikorn D, et al (2017) National and subnational populationbased incidence of cancer in Thailand: assessing cancers with the highest burdens. Cancers 9, ID 108.

6. Wiwatanadate P (2011) Lung cancer related to environmental and occupational hazards and epidemiology in Chiang Mai, Thailand. Genes Environ 33, 120-127.
7. Chuesaard T, Chetiyanukornkul T, Kameda T, Hayakawa K, Toriba A (2014) Influence of biomass burning on the levels of atmospheric polycyclic aromatic hydrocarbons and their nitro derivatives in Chiang Mai, Thailand. Aerosol Air Qual Res 14, 1247-1257.

8. Pengchai P, Chantara S, Sopajaree K, Wangkarn S, Tengcharoenkul U, Rayanakorn M (2009) Seasonal variation, risk assessment and source estimation of PM10 and PM10-bound PAHs in the ambient air of Chiang Mai and Lamphun, Thailand. Environ Monit Assess 154, 197-218.

9. Orakij W, Chetiyanukornkul T, Kasahara C, Boongla Y, Chuesaard T, Furuuchi M, Hata M, Tang N, et al (2017) Polycyclic aromatic hydrocarbons and their nitro derivatives from indoor biomass-fueled cooking in two rural areas of Thailand: a case study. Air Qual Atmos Health 10, 747-761.

10. Bumroongkit K, Rannala B, Traisathit P, Srikummool M, Wongchai Y, Kangwanpong D (2008) TP53 gene mutations of lung cancer patients in upper northern Thailand and environmental risk factors. Cancer Genet Cytogenet 185, 20-27.

11. Autsavapromporn N, Klunklin P, Threeratana C, Tuntiwechapikul W, Hosoda M, Tokonami S (2018) Short telomere length as a biomarker risk of lung cancer development induced by high radon levels: a pilot study. Int J Environ Res Public Health 15, ID 2152.

12. Klinchid J, Chewaskulyoung B, Saeteng S, Lertprasertsuke N, Kasinrerk W, Cressey R (2009) Effect of combined genetic polymorphisms on lung cancer risk in northern Thai women. Cancer Genet Cytogenet 195, 143-149.

13. Choi YY, Kang HK, Choi JE, Jang JS, Kim EJ, Cha SI, Lee WK, Kam S, et al (2008) Comprehensive assessment of P21 polymorphisms and lung cancer risk. J Hum Genet 53, 87-95.

14. Qiuling S, Yuxin Z, Suhua Z, Cheng X, Shuguang L, Fengsheng H (2003) Cyclin D1 gene polymorphism and susceptibility to lung cancer in a Chinese population. Carcinogenesis 24, 1499-1503.

15. Zhang X, Miao X, Guo Y, Tan W, Zhou Y, Sun T, Wang Y, Lin D (2006) Genetic polymorphisms in cell cycle regulatory genes MDM2 and TP53 are associated with susceptibility to lung cancer. Hum Mutat 27, 110-117.

16. Zhang X, Miao X, Sun T, Tan W, Qu S, Xiong P, Zhou Y, Lin D (2005) Functional polymorphisms in cell death pathway genes FAS and FASL contribute to risk of lung cancer. $J$ Med Genet 42, 479-484.

17. Park KH, Lo Han SG, Whang YM, Lee HJ, Yoo YD, Lee JW, Shin SW, Kim YH (2006) Single nucleotide polymorphisms of the TGFB1 gene and lung cancer risk in a Korean population. Cancer Genet Cytogenet 169, 39-44.

18. Ren Y, Yin Z, Li K, Wan Y, Li X, Wu W, Guan P, Zhou B (2015) TGF $\beta-1$ and TGFBR2 polymorphisms, 
cooking oil fume exposure and risk of lung adenocarcinoma in Chinese nonsmoking females: a case control study. BMC Med Genet 16, ID 22.

19. Ren YW, Yin ZH, Wan Y, Guan P, Wu W, Li XL, Zhou BS (2013) P53 Arg72Pro and MDM2 SNP309 polymorphisms cooperate to increase lung adenocarcinoma risk in Chinese female non-smokers: a case control study. Asian Pac J Cancer Prev 14, 5415-5420.

20. Seielstad M, Bekele E, Ibrahim M, Touré A, Traoré M (1999) A view of modern human origins from Y chromosome microsatellite variation. Genome Res $\mathbf{9}$, 558-567.

21. Hishida A, Matsuo K, Tajima K, Ogura M, Kagami Y, Taji H, Morishima Y, Emi N, et al (2004) Polymorphisms of p53 Arg72Pro, p73 G4C14-to-A4T14 at exon 2 and p21 Ser31Arg and the risk of nonHodgkin's lymphoma in Japanese. Leuk Lymphoma 45, 957-964.

22. Hashemi M, Fazaeli A, Ghavami S, Eskandari-Nasab E, Arbabi F, Mashhadi MA, Taheri M, Chaabane W, et al (2013) Functional polymorphisms of FAS and FASL gene and risk of breast cancer - pilot study of 134 cases. PLoS One 8, e53075.

23. Heidari Z, Mahmoudzadeh-Sagheb H, Rigi-Ladiz MA, Taheri M, Moazenni-Roodi A, Hashemi M (2013) Association of TGF- $\beta 1-509$ C/T, 29 C/T and 788 C/T gene polymorphisms with chronic periodontitis: a case-control study. Gene 518, 330-334.

24. Collins A, Ke X (2012) Primer1: primer design web service for tetra-primer ARMS-PCR. Open Bioinfo $J 6$, 55-58.

25. Fan H, Yu H, Deng H, Chen X (2014) Transforming growth factor- $\beta 1$ rs 1800470 polymorphism is associated with lung cancer risk: a meta-analysis. Med Sci Monit 20, 2358-2362.

26. Kang HG, Chae MH, Park JM, Kim EJ, Park JH, Kam S, Cha SI, Kim CH, et al (2006) Polymorphisms in TGF- $\beta 1$ gene and the risk of lung cancer. Lung Cancer 52, 1-7.

27. Martelossi Cebinelli GC, Paiva Trugilo K, Badaró Garcia S, Brajão de Oliveira K (2016) TGF- $\beta 1$ functional polymorphisms: a review. Eur Cytokine Netw 27, 81-89.

28. Deepthi G, Chaithri PK, Latha P, Rani VU, Rahman PF, Jahan P (2015) TGFB1 functional gene polymor- phisms (C-509T and T869C) in the maternal susceptibility to pre-eclampsia in south Indian women. Scand J Immunol 82, 390-397.

29. Haeger SM, Thompson JJ, Kalra S, Cleaver TG, Merrick D, Wang XJ, Malkoski SP (2016) Smad4 loss promotes lung cancer formation but increases sensitivity to DNA topoisomerase inhibitors. Oncogene 35, 577-586.

30. Zhao M, Mishra L, Deng CX (2018) The role of TGF- $\beta$ /SMAD4 signaling in cancer. Int $J$ Biol Sci 14, 111-123.

31. Mu L, Liu L, Niu R, Zhao B, Shi J, Li Y, Swanson M, Scheider W, et al (2013) Indoor air pollution and risk of lung cancer among Chinese female non-smokers. Cancer Causes Control 24, 439-450.

32. Chang LW, Lo WS, Lin P (2005) Trans, trans-2,4decadienal, a product found in cooking oil fumes, induces cell proliferation and cytokine production due to reactive oxygen species in human bronchial epithelial cells. Toxicol Sci 87, 337-343.

33. Lerrer S, Liubomirski Y, Bott A, Abnaof K, Oren N, Yousaf A, Körner C, Meshel T, et al (2017) Coinflammatory roles of TGF $\beta 1$ in the presence of TNF $\alpha$ drive a pro-inflammatory fate in mesenchymal stem cells. Front Immunol 8, ID 479.

34. Pungkhom P, Jinsart W (2014) Health risk assessment from bush fire air pollutants using statistical analysis and geographic information system: a case study in northern Thailand. Int J Geoinfo 10, 17-24.

35. Sirimongkonlertkul N, Upayokhin P, Phonekeo V (2013) Multi-temporal analysis of haze problem in northern Thailand: a case study in Chiang Rai province. Agric Nat Resour 47, 768-780.

36. Hamra GB, Guha N, Cohen A, Laden F, RaaschouNielsen O, Samet JM, Vineis P, Forastiere F, et al (2014) Outdoor particulate matter exposure and lung cancer: a systematic review and meta-analysis. Environ Health Perspect 122, 906-911.

37. Kellar SP, Kelvin EA (2013) Munro's Statistical Methods for Health Care Research, 6th edn, Lippincott Williams \& Wilkins, Philadelphia, USA.

38. Fletcher RH, Fletcher SW, Fletcher GS (2014) Clinical Epidemiology: the Essentials, 5th edn, Lippincott Williams \& Wilkins, Philadelphia, USA. 


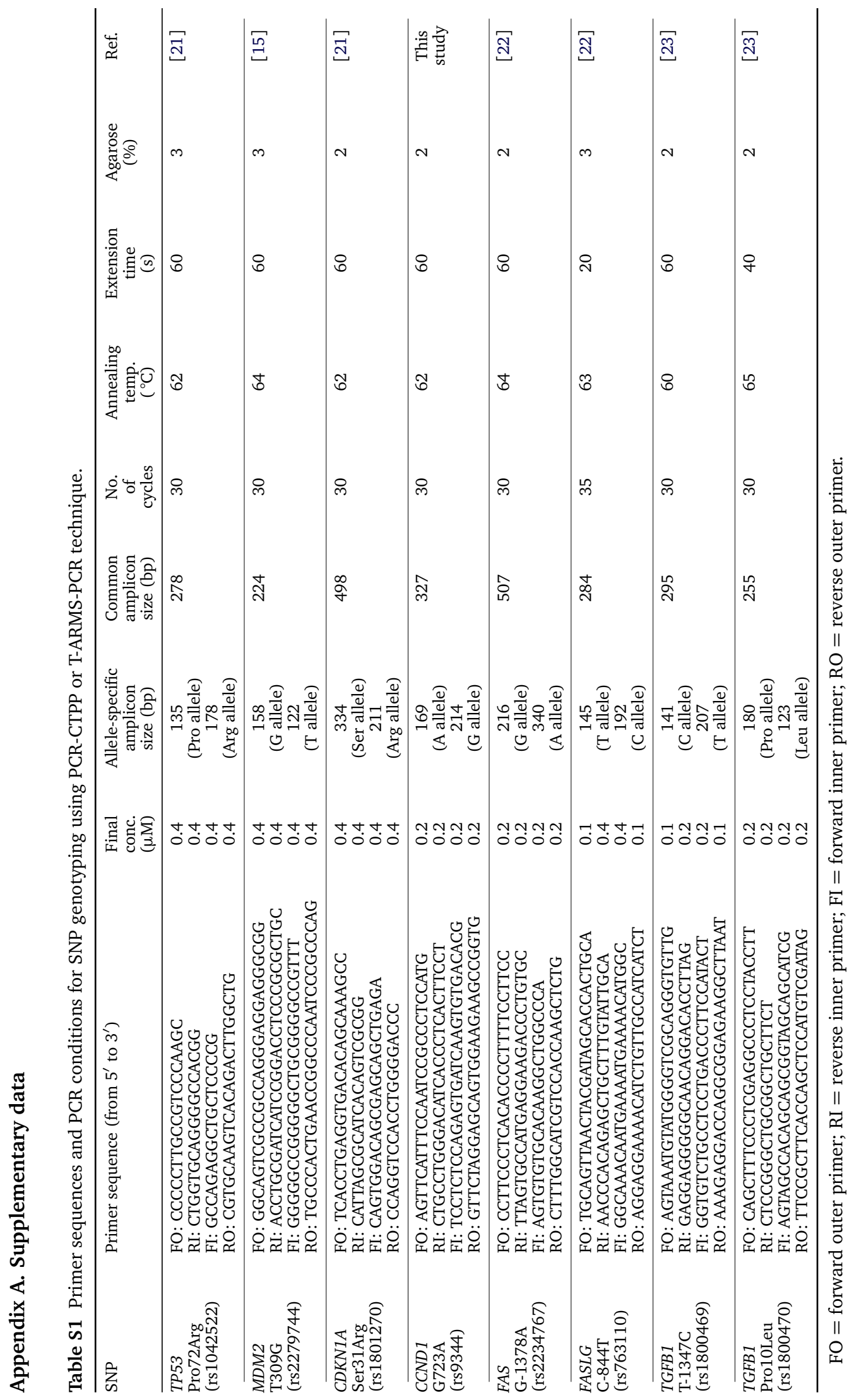

